\title{
Determination of stress and displacement state in the circular annulus steel sheet with Cartesian orthotropy
}
F. Kosel ${ }^{1}$
V. Bratuš ${ }^{2}$
T. Kosel ${ }^{3}$
T. Videnič ${ }^{4}$

(Received 1 September 2006; revised 16 February 2010)

\begin{abstract}
This paper deals with determination of stress, strain and displacement state of a circular annulus which is made of material with Cartesian orthotropic rheological behaviour. There is a continuous and constant load on the inner and/or outer edge. Stress and strain state in the circular annulus is in the elastic domain. Stress state is determined on the basis of an Airy stress function, where all boundary conditions have to be fulfilled. The Ritz's method was applied to determine unknown parameters of the Airy function.
\end{abstract}

\section{Contents}


3 Transformation of Hooke's law of orthotropic sheet steel C1134

4 Stress, strain and displacement state in the circular annulus

C1136

4.1 Ritz's method . . . . . . . . . . . . . . . . C1139

4.2 Non-homogeneous equations system . . . . . . . . . . . C1141

5 Numerical results

C1143

6 Conclusion

C1147

References

C1148

\section{Introduction}

In high volume punching and further processing of steel sheet components, engineers are often faced with the problem of having to meet very narrow dimensional and geometrical tolerances in order to satisfy customer's needs. When the allowable tolerance range is only ten micrometers or so, this requires very accurate adjustment of all technological parameters of the metal working process. For this reason the adjustment of process parameters should be based on prior analyses of stress-strain states in the material. Only in this way can the required quality of the end product be achieved.

Steel sheet is a typical plane structure element whose orthotropy is defined by two directions: the direction of rolling $x_{1}$ and the direction perpendicular to the direction of rolling $x_{2}$. For solving the stress, strain and displacement states in products punched from steel sheet into a round shape or circular annulus the cylindrical coordinate system was used. By measurements of Cartesian orthotropy of the steel sheet and by transforming all elastic constants of the generalized Hooke's law into a cylindrical coordinate system, it is possible to define a mathematical computational model for the circular annulus. The stress, strain and displacement states of products having the 
shape of circular annulus can then be defined accurately enough by the Airy stress function [1]. On the basis of accurate results of stress and strain states it should be possible to determine the metal working parameters even for highly demanding products of complex shapes and narrow tolerance ranges.

In the available literature the treatment of the circular annulus with Cartesian orthotropy is quite rare. Some works have been published on the treatment of vibrations of such annulus [2]. Research where rectangular plates with Cartesian orthotropy are dealt with are much easier to find $[3,4,5,6]$.

\section{Hooke's law of orthotropic body}

The general relationship between the components of the stress and strain tensors for anisotropic steel sheet [7] can be written in the form of a fourth order tensors $a_{i j k l}$ and $A_{i j k l}$ :

$$
\varepsilon_{i j}=a_{i j k l} \sigma_{k l} \quad \text { or } \quad \sigma_{i j}=A_{i j k l} \varepsilon_{k l},
$$

where $\varepsilon_{i j}$ is the strain tensor, $\sigma_{i j}$ is the stress tensor, $a_{i j k l}$ and $A_{i j k l}$ are tensors of moduli of elasticity. In the case of Cartesian orthogonal coordinate system $\left(x_{1}, x_{2}, x_{3}\right)$ the subscripts $\mathbf{i}, \mathbf{j}, \mathbf{k}, \mathbf{l}$ take values $1,2,3$. The following symmetry holds: $a_{i j k l}=a_{k l i j}$ and $A_{i j k l}=A_{k l i j}$. Hooke's law of a $3 \mathrm{D}$ orthotropic body in matrix form is $\{\varepsilon\}=[\overline{\mathbf{a}}]\{\sigma\}$, that is,

$$
\left\{\begin{array}{c}
\varepsilon_{11} \\
\varepsilon_{22} \\
\varepsilon_{33} \\
\varepsilon_{23} \\
\varepsilon_{31} \\
\varepsilon_{12}
\end{array}\right\}=\left[\begin{array}{cccccc}
\overline{\mathrm{a}}_{11} & \overline{\mathrm{a}}_{12} & \overline{\mathrm{a}}_{13} & 0 & 0 & 0 \\
& \overline{\mathrm{a}}_{22} & \overline{\mathrm{a}}_{23} & 0 & 0 & 0 \\
& & \overline{\mathrm{a}}_{33} & 0 & 0 & 0 \\
& & & \overline{\mathrm{a}}_{44} & 0 & 0 \\
& & & & \overline{\mathrm{a}}_{55} & 0 \\
& & & & & \overline{\mathrm{a}}_{66}
\end{array}\right\}\left\{\begin{array}{c}
\sigma_{11} \\
\sigma_{22} \\
\sigma_{33} \\
\sigma_{23} \\
\sigma_{31} \\
\sigma_{12}
\end{array}\right\}
$$

where double elastic symmetry is considered [7]. The elements of the matrix of elastic constants $[\overline{\mathbf{a}}]$ are functions of the engineering constants, $\mathrm{E}_{\boldsymbol{i}}$, Young's 
modulus in direction $i, G_{i j}$, the shear modulus and $v_{i j}$, Poisson's ratio, which is the contraction of body in $\boldsymbol{j}$ direction due to the force acting in $\boldsymbol{i}$ direction. Equation (2) is equivalent to

$$
\begin{aligned}
& \varepsilon_{11}=\frac{1}{E_{1}} \sigma_{11}-\frac{v_{21}}{E_{2}} \sigma_{22}-\frac{v_{31}}{E_{3}} \sigma_{33}, \\
& \varepsilon_{22}=-\frac{v_{12}}{E_{1}} \sigma_{11}+\frac{1}{E_{2}} \sigma_{22}-\frac{v_{32}}{E_{3}} \sigma_{33}, \\
& \varepsilon_{33}=-\frac{v_{13}}{E_{1}} \sigma_{11}-\frac{v_{23}}{E_{2}} \sigma_{22}+\frac{1}{E_{3}} \sigma_{33}, \\
& \varepsilon_{23}=\frac{1}{2 G_{23}} \sigma_{23}, \\
& \varepsilon_{31}=\frac{1}{2 G_{31}} \sigma_{31}, \\
& \varepsilon_{12}=\frac{1}{2 G_{12}} \sigma_{12} .
\end{aligned}
$$

Because of the symmetry of stress and strain tensor, the following equality must hold:

$$
E_{i} v_{j i}=E_{j} v_{i j}, \quad i, j=1,2,3 .
$$

\section{Transformation of Hooke's law of orthotropic sheet steel into the cylindrical coordinate system}

The generalized Hooke's law in the Cartesian coordinate system is defined by Equation (1). The elastic strain energy for the whole body, $W$, has to remain unchanged in a new coordinate system $\left(x_{1}^{\prime}, x_{2}^{\prime}, x_{3}^{\prime}\right)$ since $W$ is an invariant of the coordinate system. Thus,

$$
W=\frac{1}{2} \int \sigma_{i j} \varepsilon_{i j} d V=\frac{1}{2} \int \sigma_{i j}^{\prime} \varepsilon_{i j}^{\prime} d V^{\prime} .
$$


The relationship between both coordinate systems is expressed with the transformation tensor $\alpha_{i j}$. Consider a case when a new coordinate system is obtained from the old one $\left(x_{1}, x_{2}, x_{3}\right)$ by rotating it about the common axis $x_{3}=x_{3}^{\prime}$ by an angle $\varphi$. The transformation tensor $\alpha_{i j}$ in this case is

$$
\alpha_{i j}=\left(\begin{array}{ccc}
\cos \varphi & \sin \varphi & 0 \\
-\sin \varphi & \cos \varphi & 0 \\
0 & 0 & 1
\end{array}\right)
$$

According to tensor transformation law, the stress tensor in a new coordinate system $\left(x_{1}^{\prime}, x_{2}^{\prime}, x_{3}^{\prime}\right)$ is

$$
\sigma_{i j}^{\prime}=\alpha_{i k} \alpha_{j l} \sigma_{k l} .
$$

Further, Hooke's law of orthotropic body in a matrix form is expressed by

$$
\left\{\varepsilon^{\prime}\right\}=\left[\overline{\mathbf{a}}^{\prime}\right]\left\{\boldsymbol{\sigma}^{\prime}\right\}=[\mathbf{q}][\overline{\mathbf{a}}][\mathbf{q}]^{\top}\left\{\sigma^{\prime}\right\}, \quad\left[\overline{\mathbf{a}}^{\prime}\right]=[\mathbf{q}][\overline{\mathbf{a}}][\mathbf{q}]^{\top},
$$

where $[\mathbf{q}]$ is a transformation matrix [7]. The superscript $T$ indicates the transpose operation. By this process each coefficient of the matrix of elastic constant $\left[\overline{\mathbf{a}}^{\prime}\right]$ in a new coordinate system is explicitly defined.

By using 'engineering constants' $E_{i}, G_{i j}$ and $v_{i j}$ in the cylindrical coordinate system and taking into consideration Equation (4), the final transformation equations [7] are

$$
\begin{aligned}
& \overline{\mathrm{a}}_{11}^{\prime}=\frac{\cos ^{4} \varphi}{\mathrm{E}_{1}}+\left(\frac{1}{\mathrm{G}_{12}}-\frac{2 v_{12}}{\mathrm{E}_{1}}\right) \sin ^{2} \varphi \cos ^{2} \varphi+\frac{\sin ^{4} \varphi}{\mathrm{E}_{2}}, \\
& \overline{\mathrm{a}}_{22}^{\prime}=\frac{\sin ^{4} \varphi}{\mathrm{E}_{1}}+\left(\frac{1}{\mathrm{G}_{12}}-\frac{2 v_{12}}{\mathrm{E}_{1}}\right) \sin ^{2} \varphi \cos ^{2} \varphi+\frac{\cos ^{4} \varphi}{\mathrm{E}_{2}}, \\
& \overline{\mathrm{a}}_{12}^{\prime}=\left(\frac{1}{\mathrm{E}_{1}}+\frac{1}{\mathrm{E}_{2}}+\frac{2 v_{12}}{\mathrm{E}_{1}}-\frac{1}{\mathrm{G}_{12}}\right) \sin ^{2} \varphi \cos ^{2} \varphi-\frac{v_{12}}{\mathrm{E}_{1}}, \\
& \overline{\mathrm{a}}_{66}^{\prime}=4\left(\frac{1}{\mathrm{E}_{1}}+\frac{1}{\mathrm{E}_{2}}+\frac{2 v_{12}}{\mathrm{E}_{1}}-\frac{1}{\mathrm{G}_{12}}\right) \sin ^{2} \varphi \cos ^{2} \varphi+\frac{1}{\mathrm{G}_{12}}, \\
& \overline{\mathrm{a}}_{16}^{\prime}=\left[\left(\frac{\sin ^{2} \varphi}{\mathrm{E}_{2}}-\frac{\cos ^{2} \varphi}{\mathrm{E}_{1}}\right)+\left(\frac{1}{2 \mathrm{G}_{12}}-\frac{v_{12}}{\mathrm{E}_{1}}\right) \cos 2 \varphi\right] \sin 2 \varphi,
\end{aligned}
$$




$$
\overline{\mathrm{a}}_{26}^{\prime}=\left[\left(\frac{\cos ^{2} \varphi}{\mathrm{E}_{2}}-\frac{\sin ^{2} \varphi}{\mathrm{E}_{1}}\right)-\left(\frac{1}{2 \mathrm{G}_{12}}-\frac{v_{12}}{\mathrm{E}_{1}}\right) \cos 2 \varphi\right] \sin 2 \varphi .
$$

Also elements $\overline{\mathrm{a}}_{44}^{\prime}, \overline{\mathrm{a}}_{45}^{\prime}, \overline{\mathrm{a}}_{55}^{\prime}, \overline{\mathrm{a}}_{13}^{\prime}, \overline{\mathrm{a}}_{23}^{\prime}, \overline{\mathrm{a}}_{33}^{\prime}$ and $\overline{\mathrm{a}}_{36}^{\prime}$ are not equal to zero. The geometry of the steel sheet body can be considered as a plane problem with plane stress state. Thus the displacement state in the circular annulus determined without knowing all of them precisely. The other coefficients of the matrix are equal to zero [7].

\section{Determination of stress, strain and displacement state in the circular annulus}

Figure 1 shows the orthotropic circular annulus made from material with Cartesian orthotropy, Young's modulus $E_{1}, E_{2}$, and continuous load $p_{n}$ on the inner and $p_{z}$ on the outer edge. The cylindrical coordinate system has to be applied because of the axi-symmetric geometry. As already mentioned, the steel sheet body is considered as a plane stress state problem only. The relation between elements of the stress tensor $\sigma_{r}, \sigma_{\phi}$ and $\tau_{\mathrm{r} \phi}$ and elements of the strain tensor $\mathcal{E}_{\mathrm{r}}, \varepsilon_{\varphi}$ and $\gamma_{\mathrm{r} \varphi}$ is written in the cylindrical coordinate system as

$$
\begin{aligned}
\varepsilon_{r}= & \bar{a}_{11}^{\prime} \sigma_{r}+\bar{a}_{12}^{\prime} \sigma_{\phi}+\bar{a}_{16}^{\prime} \tau_{r \phi}=\frac{\partial u_{r}}{\partial r}, \\
\varepsilon_{\phi} & =\bar{a}_{12}^{\prime} \sigma_{r}+\bar{a}_{22}^{\prime} \sigma_{\phi}+\bar{a}_{26}^{\prime} \tau_{r \phi}=\frac{1}{r} \frac{\partial u_{\phi}}{\partial \phi}+\frac{u_{r}}{r}, \\
\gamma_{r \phi} & =2 \varepsilon_{r \phi}=\bar{a}_{16}^{\prime} \sigma_{r}+\bar{a}_{26}^{\prime} \sigma_{\phi}+\bar{a}_{66}^{\prime} \tau_{r \phi} \\
& =\frac{1}{r} \frac{\partial u_{r}}{\partial r}+\frac{\partial u_{\phi}}{\partial r}-\frac{u_{\phi}}{r},
\end{aligned}
$$

with $\mathfrak{u}_{\boldsymbol{r}}$ and $\mathfrak{u}_{\varphi}$ displacements in radial and circular direction respectively.

The stress tensor in the cylindrical coordinate system is expressed by the Airy stress function. The relationships between the elements of stress tensor 


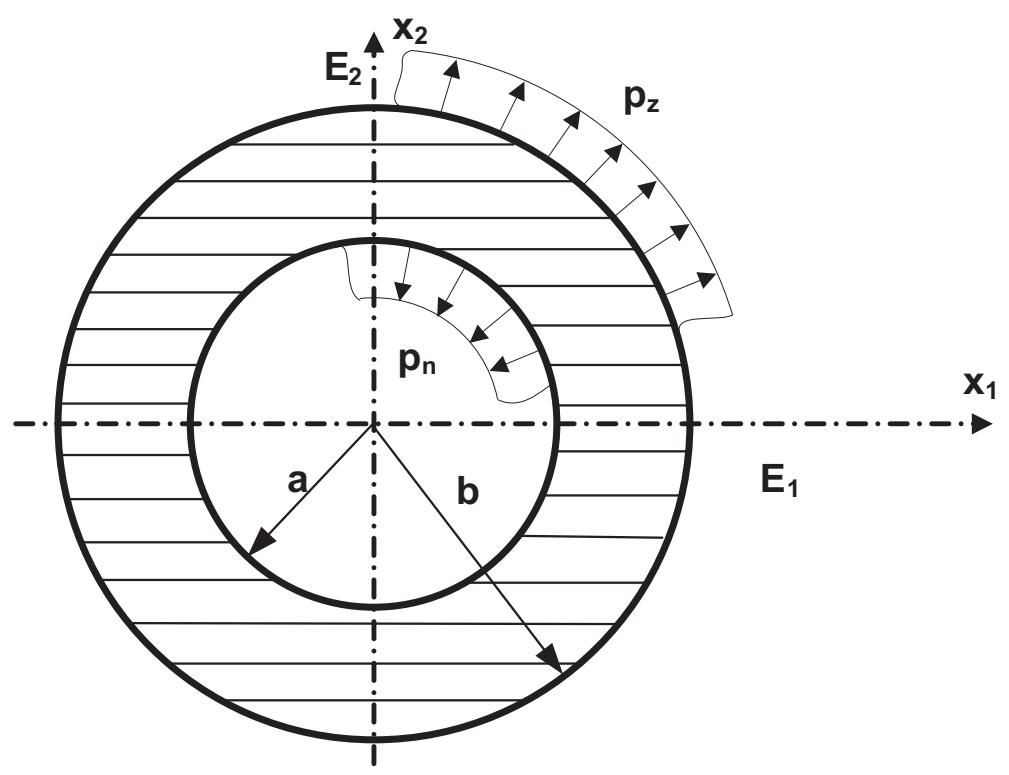

Figure 1: Circular annulus with Cartesian orthotropy. 
and the Airy function F, neglecting the mass force, are well known from the theory of elasticity

$$
\sigma_{r}=\frac{1}{r} \frac{\partial F}{\partial r}+\frac{1}{r^{2}} \frac{\partial^{2} F}{\partial \varphi^{2}}, \quad \sigma_{\varphi}=\frac{\partial^{2} F}{\partial r^{2}}, \quad \tau_{r \varphi}=-\frac{\partial^{2}}{\partial r \partial \varphi}\left(\frac{F}{r}\right) .
$$

The elastic strain energy is given by Equation (5), where integration in the direction of $z$ axis, that is, thickness of the circular annulus within limits $|z| \leqslant$ $\mathrm{h} / 2$, has to be done. Introducing Equation (10) and (11) into Equation (5) yields

$$
\begin{aligned}
W= & \frac{h}{2} \int_{A} \sigma_{i j} \varepsilon_{i j} d A \\
= & \frac{h}{2} \int_{A}\left\{\left[\bar{a}_{11}^{\prime}\left(\frac{1}{r} \frac{\partial F}{\partial r}+\frac{1}{r^{2}} \frac{\partial^{2} \mathrm{~F}}{\partial \phi^{2}}\right)^{2}+\bar{a}_{22}^{\prime}\left(\frac{\partial^{2} \mathrm{~F}}{\partial r^{2}}\right)^{2}\right.\right. \\
& \left.\left.+\bar{a}_{66}^{\prime}\left(-\frac{\partial^{2}}{\partial r \partial \phi}\left(\frac{F}{r}\right)\right)^{2}\right]\right\}+2 \bar{a}_{12}^{\prime}\left(\frac{1}{r} \frac{\partial F}{\partial r}+\frac{1}{r^{2}} \frac{\partial^{2} \mathrm{~F}}{\partial \phi^{2}}\right)\left(\frac{\partial^{2} \mathrm{~F}}{\partial \mathrm{r}^{2}}\right) \\
& +2 \overline{\mathrm{a}}_{16}^{\prime}\left(\frac{1}{\mathrm{r}} \frac{\partial \mathrm{F}}{\partial \mathrm{r}}+\frac{1}{\mathrm{r}^{2}} \frac{\partial^{2} \mathrm{~F}}{\partial \phi^{2}}\right)\left(-\frac{\partial^{2}}{\partial r \partial \phi}\left(\frac{\mathrm{F}}{\mathrm{r}}\right)\right) \\
& +2 \overline{\mathrm{a}}_{26}^{\prime}\left\{\left(\frac{\partial^{2} \mathrm{~F}}{\partial \mathrm{r}^{2}}\right)\left(-\frac{\partial^{2}}{\partial r \partial \phi}\left(\frac{\mathrm{F}}{\mathrm{r}}\right)\right)\right\} \mathrm{d} A .
\end{aligned}
$$

The choice of the Airy stress function depends on the body's shape, the way of loading, and the boundary conditions. In the case of a circular annulus with a constant and continuous load on the inner and outer edge, Figure 1, the boundary conditions are $\sigma_{\mathrm{r}}(\mathrm{a})=\mathrm{p}_{\mathrm{n}}$ and $\sigma_{\mathrm{r}}(\mathrm{b})=\mathrm{p}_{z}$ and the Airy stress function applied in the following form [1]:

$$
\begin{aligned}
F(r, \varphi)= & -\left(p_{z}-p_{n}\right) \frac{a^{2} b^{2}}{b^{2}-a^{2}} \ln r-\frac{r^{2}}{2} \frac{\left(p_{n} a^{2}-p_{z} b^{2}\right)}{b^{2}-a^{2}} \\
& +\left(1-\frac{r^{2}}{b^{2}}\right)^{2}\left(\frac{r^{2}}{a^{2}}-1\right) \sum_{k=1}^{k=n} \alpha_{k}\left(\frac{r}{b}\right)^{2 k} \cos 2 k \varphi
\end{aligned}
$$




$$
=f_{0}+\alpha_{k} f_{k}
$$

The stress function in Equation (13) contains coefficients $\alpha_{k}$ which have to be defined. Ritz's method determined them in this research.

\subsection{Ritz's method}

The theorem of the condition of minimum strain energy states that strain energy of an elastic system in the equilibrium state has an extreme which is guaranteed to be a minimum. Coefficients $\alpha_{k}$ represent constants and can be defined on the basis of the condition of minimum strain energy. The elastic strain energy function has an extreme, when the partial differentiations with respect to coefficients $\alpha_{k}$ are equal to zero:

$$
\frac{\partial W}{\partial \alpha_{k}}=0, \quad k=1, \ldots, n .
$$

According to equations (12) and (13), equations (14) represents a non-homogenec system of $n$ linear equations with $n$ unknowns. By solving this system and getting values for coefficients $\alpha_{k}$, the Airy function is finally completed.

With introduction of functions $G_{i}\left(\alpha_{k}, r, \varphi\right), i=1,2,3$, which contain the functions of differentiations of Airy stress function in the general elastic strain energy Equation (12), the following relations are obtained:

$$
\begin{aligned}
\mathrm{G}_{1}\left(\alpha_{\mathrm{k}}, \mathrm{r}, \varphi\right)=\frac{1}{\mathrm{r}} \frac{\partial \mathrm{F}}{\partial \mathrm{r}}+\frac{1}{\mathrm{r}^{2}} \frac{\partial^{2} \mathrm{~F}}{\partial \varphi^{2}} \\
=-\left(\mathrm{p}_{z}-\mathrm{p}_{\mathrm{n}}\right) \frac{\mathrm{a}^{2} \mathrm{~b}^{2}}{\left(\mathrm{~b}^{2}-\mathrm{a}^{2}\right) \mathrm{r}^{2}}-\frac{\left(\mathrm{p}_{\mathrm{n}} \mathrm{a}^{2}-\mathrm{p}_{z} \mathrm{~b}^{2}\right)}{\mathrm{b}^{2}-\mathrm{a}^{2}} \\
-4\left(1-\frac{\mathrm{r}^{2}}{\mathrm{~b}^{2}}\right)\left(\frac{\mathrm{r}^{2}}{\mathrm{a}^{2}} 1\right)\left[\frac{1}{\mathrm{~b}^{2}}\left(\frac{\mathrm{r}^{2}}{\mathrm{a}^{2}}-1\right)-\frac{1}{\mathrm{a}^{2}}\left(1-\frac{\mathrm{r}^{2}}{\mathrm{~b}^{2}}\right)\right] \\
\quad \times \sum_{k=1}^{k=n} \alpha_{k}\left(\frac{r}{b}\right)^{2 k} \cos 2 k \varphi+\frac{1}{r}\left(1-\frac{\mathrm{r}^{2}}{\mathrm{~b}^{2}}\right)^{2}\left(\frac{\mathrm{r}^{2}}{\mathrm{a}^{2}}-1\right)^{2}
\end{aligned}
$$




$$
\begin{aligned}
& \times\left[\sum_{k=1}^{k=n} \alpha_{k} \frac{2 k}{b}\left(\frac{r}{b}\right)^{2 k-1} \cos 2 k \varphi-\frac{1}{r} \sum_{k=1}^{k=n} 4 k^{2} \alpha_{k}\left(\frac{r}{b}\right)^{2 k} \cos 2 k \varphi\right] \\
& G_{3}\left(\alpha_{k}, r, \varphi\right)=-\frac{\partial^{2}}{\partial r \partial \varphi}\left(\frac{F}{r}\right) \\
&= \frac{1}{r}\left(1-\frac{r^{2}}{b^{2}}\right)^{2}\left(\frac{r^{2}}{a^{2}}-1\right) \sum_{k=1}^{2 k=n} \alpha_{k} \frac{4 k^{2}}{b}\left(\frac{r}{b}\right)^{2 k-1} \sin 2 k \varphi \\
&+\left(1-\frac{r^{2}}{b^{2}}\right)\left(\frac{r^{2}}{a^{2}}-1\right)\left[\left(1-\frac{r^{2}}{b^{2}}\right)\left(\frac{3}{a^{2}}+\frac{1}{r^{2}}\right)-\frac{4}{b^{2}}\left(\frac{r^{2}}{a^{2}}-1\right)\right] \\
& \times \sum_{k=1}^{k=n} 2 k \alpha_{k}\left(\frac{r}{b}\right)^{2 k} \sin 2 k \varphi \\
& G_{2}\left(\alpha_{k}, r, \varphi\right)=\frac{\partial^{2} F}{\partial r^{2}} \\
&=-\left(p_{n}-p_{z}\right) \frac{a^{2} b^{2}}{\left(b^{2}-a^{2}\right) r^{2}}-\frac{\left(p_{n} a^{2}-p_{z} b^{2}\right)}{b^{2}-a^{2}}-\frac{4 r}{b^{2}}\left(1-\frac{r^{2}}{b^{2}}\right) \\
& \times\left(\frac{r^{2}}{a^{2}}-1\right)^{2} \sum_{k=1}^{k=n} \alpha_{k} \frac{2 k}{b}\left(\frac{r}{b}\right)^{2 k-1} \cos 2 k \varphi+\left(1-\frac{r^{2}}{b^{2}}\right)^{2} \\
& \times\left(\frac{r^{2}}{a^{2}}-1\right)^{2} \sum_{k=1}^{k=n} \alpha_{k} \frac{2 k(2 k-1)}{b^{2}}\left(\frac{r}{b}\right)^{2 k-2} \cos 2 k \varphi \\
&+4 r\left(1-\frac{r^{2}}{b^{2}}\right)\left(\frac{r^{2}}{a^{2}}-1\right)\left[\frac{2}{a^{2}}\left(1-\frac{r^{2}}{b^{2}}\right)-\frac{1}{b^{2}}\left(\frac{r^{2}}{a^{2}}-1\right)\right] \\
& \times \sum_{k=1}^{k} \alpha_{k} \frac{2 k}{b}\left(\frac{r}{b}\right)^{2 k-1} \cos 2 k \varphi-4 \sum_{k=1}^{2} \alpha_{k}\left(\frac{r}{b}\right)^{2 k} \cos 2 k \varphi \\
& \times\left[b^{2}\left(3 b^{2}\left(5 r^{2}-b^{2}\right)-14 r^{4}\right)+a^{4}\left(b^{2}-3 r^{2}\right)\right. \\
& a^{4} b^{4}
\end{aligned}
$$


Equations (14), with reference to equations (15), and additionally introducing functional dependence $\overline{\mathbf{a}}_{i j}^{\prime}=\overline{\mathrm{a}}_{\mathfrak{i j}}^{\prime}(\varphi)$, takes the following form:

$$
\begin{aligned}
\frac{\partial W}{\partial \alpha_{k}}= & \frac{h}{2} \int_{\mathcal{A}}\left\{2 \overline{\mathbf{a}}_{11}^{\prime}(\varphi) G_{1} \frac{\partial G_{1}}{\partial \alpha_{k}}+2 \bar{a}_{22}^{\prime}(\varphi) G_{2} \frac{\partial G_{2}}{\partial \alpha_{k}}+2 \bar{a}_{66}^{\prime}(\varphi) G_{3} \frac{\partial G_{3}}{\partial \alpha_{k}}\right. \\
& +2 \bar{a}_{12}^{\prime}(\varphi)\left(\frac{\partial G_{1}}{\partial \alpha_{k}} G_{2}+G_{1} \frac{\partial G_{2}}{\partial \alpha_{k}}\right)+2 \bar{a}_{16}^{\prime}(\varphi)\left(\frac{\partial G_{1}}{\partial \alpha_{k}} G_{3}+G_{1} \frac{\partial G_{3}}{\partial \alpha_{k}}\right) \\
& \left.+2 \bar{a}_{26}^{\prime}(\varphi)\left(\frac{\partial G_{2}}{\partial \alpha_{k}} G_{3}+G_{2} \frac{\partial G_{3}}{\partial \alpha_{k}}\right)\right\} d A=0 .
\end{aligned}
$$

Moreover, by introducing the functions $\overline{\mathrm{G}}_{\mathrm{ki}}(\mathrm{r}, \varphi)$ which are dependent on coordinates $r$ and $\varphi$, equations (15) becomes

$$
\mathrm{G}_{\mathrm{i}}\left(\alpha_{\mathrm{k}}, \mathrm{r}, \varphi\right)=\mathrm{c}_{\mathrm{i}}(\mathrm{r})+\sum_{\mathrm{k}=1}^{\mathrm{k}=\mathrm{n}} \alpha_{\mathrm{k}} \overline{\mathrm{G}}_{\mathrm{ki}}(\mathrm{r}, \varphi), \quad \boldsymbol{i}=1,2,3 .
$$

Coefficients $c_{\mathfrak{i}}(r)$ represent factors not being multiplied by $\alpha_{k}$. Partial differentiations of function $G_{i}$ with respect to coefficients $\alpha_{k}$ are thus

$$
\frac{\partial G_{i}\left(\alpha_{k}, r, \varphi\right)}{\partial \alpha_{k}}=\frac{\partial\left[c_{i}(r)+\sum_{k=1}^{k=n} \alpha_{k} \bar{G}_{k i}(r, \varphi)\right]}{\partial \alpha_{k}}=\bar{G}_{k i}(r, \varphi) .
$$

Each factor $\bar{G}_{k i}(r, \varphi)$ contains a product of trigonometric function and polynomial.

\subsection{Non-homogeneous equations system}

The number of coefficients $\alpha_{k}$ in the Airy stress function is not defined specifically. A high convergence of the Airy stress function has been evaluated through several simulations of different loading conditions. The stress state accuracy is expressed with error coefficient $\varepsilon \geqslant\left|\sigma_{i j}^{k}-\sigma_{i j}^{k-1}\right|$. Stress states $\sigma_{i j}^{k}$ 
and $\sigma_{i j}^{k-1}$ are two adjacent states obtained if $k$ or $k-1$ coefficients are used. In the case of $\varepsilon=10^{-3}$, we have to use six coefficients.

For this chosen error of the Airy stress function, equations (16) represent a non-homogeneous system of six linear equations with six unknown coefficients $\alpha_{k}$. The kth equation has the form

$$
\begin{aligned}
& \alpha_{1} \int_{A}\left[2 \overline{\mathrm{a}}_{11}^{\prime} \overline{\mathrm{G}}_{11} \overline{\mathrm{G}}_{\mathrm{k} 1}+2 \overline{\mathrm{a}}_{22}^{\prime} \overline{\mathrm{G}}_{12} \overline{\mathrm{G}}_{\mathrm{k} 2}+2 \overline{\mathrm{a}}_{66}^{\prime} \overline{\mathrm{G}}_{13} \overline{\mathrm{G}}_{\mathrm{k} 3}+2 \overline{\mathrm{a}}_{12}^{\prime} \overline{\mathrm{G}}_{12} \overline{\mathrm{G}}_{\mathrm{k} 1}\right. \\
& +2 \overline{\mathrm{a}}_{12}^{\prime} \overline{\mathrm{G}}_{11} \overline{\mathrm{G}}_{\mathrm{k} 2}+2 \overline{\mathrm{a}}_{16}^{\prime} \overline{\mathrm{G}}_{13} \overline{\mathrm{G}}_{\mathrm{k} 1}+2 \overline{\mathrm{a}}_{16}^{\prime} \overline{\mathrm{G}}_{11} \overline{\mathrm{G}}_{\mathrm{k} 3}+2 \overline{\mathrm{a}}_{26}^{\prime} \overline{\mathrm{G}}_{13} \overline{\mathrm{G}}_{\mathrm{k} 2} \\
& \left.+2 \overline{\mathrm{a}}_{26}^{\prime} \overline{\mathrm{G}}_{12} \overline{\mathrm{G}}_{\mathrm{k} 3}\right] \mathrm{d} \mathrm{A}+\alpha_{2} \int_{\mathrm{A}}\left[2 \overline{\mathrm{a}}_{11}^{\prime} \overline{\mathrm{G}}_{21} \overline{\mathrm{G}}_{\mathrm{k} 1}+2 \overline{\mathrm{a}}_{22}^{\prime} \overline{\mathrm{G}}_{22} \overline{\mathrm{G}}_{\mathrm{k} 2}+2 \overline{\mathrm{a}}_{66}^{\prime} \overline{\mathrm{G}}_{23} \overline{\mathrm{G}}_{\mathrm{k} 3}\right. \\
& +2 \overline{\mathrm{a}}_{12}^{\prime} \overline{\mathrm{G}}_{22} \overline{\mathrm{G}}_{\mathrm{k} 1}+2 \overline{\mathrm{a}}_{12}^{\prime} \overline{\mathrm{G}}_{21} \overline{\mathrm{G}}_{\mathrm{k} 2}+2 \overline{\mathrm{a}}_{16}^{\prime} \overline{\mathrm{G}}_{23} \overline{\mathrm{G}}_{\mathrm{k} 1}+2 \overline{\mathrm{a}}_{16}^{\prime} \overline{\mathrm{G}}_{21} \overline{\mathrm{G}}_{\mathrm{k} 3}+2 \overline{\mathrm{a}}_{26}^{\prime} \overline{\mathrm{G}}_{23} \overline{\mathrm{G}}_{\mathrm{k} 2} \\
& \left.+2 \overline{\mathrm{a}}_{26}^{\prime} \overline{\mathrm{G}}_{22} \overline{\mathrm{G}}_{\mathrm{k} 3}\right] \mathrm{d} \mathrm{A}+\alpha_{3} \int_{\mathrm{A}}\left[2 \overline{\mathrm{a}}_{11}^{\prime} \overline{\mathrm{G}}_{31} \overline{\mathrm{G}}_{\mathrm{k} 1}+2 \overline{\mathrm{a}}_{22}^{\prime} \overline{\mathrm{G}}_{32} \overline{\mathrm{G}}_{\mathrm{k} 2}+2 \overline{\mathrm{a}}_{66}^{\prime} \overline{\mathrm{G}}_{33} \overline{\mathrm{G}}_{\mathrm{k} 3}\right. \\
& +2 \overline{\mathrm{a}}_{12}^{\prime} \overline{\mathrm{G}}_{32} \overline{\mathrm{G}}_{\mathrm{k} 1}+2 \overline{\mathrm{a}}_{12}^{\prime} \overline{\mathrm{G}}_{31} \overline{\mathrm{G}}_{\mathrm{k} 2}+2 \overline{\mathrm{a}}_{16}^{\prime} \overline{\mathrm{G}}_{33} \overline{\mathrm{G}}_{\mathrm{k} 1}+2 \overline{\mathrm{a}}_{16}^{\prime} \overline{\mathrm{G}}_{31} \overline{\mathrm{G}}_{\mathrm{k} 3}+2 \overline{\mathrm{a}}_{26}^{\prime} \overline{\mathrm{G}}_{33} \overline{\mathrm{G}}_{\mathrm{k} 2} \\
& \left.+2 \overline{\mathrm{a}}_{26}^{\prime} \overline{\mathrm{G}}_{32} \overline{\mathrm{G}}_{\mathrm{k} 3}\right] \mathrm{d} \mathrm{A}+\alpha_{4} \int_{\mathrm{A}}\left[2 \overline{\mathrm{a}}_{11}^{\prime} \overline{\mathrm{G}}_{41} \overline{\mathrm{G}}_{\mathrm{k} 1}+2 \overline{\mathrm{a}}_{22}^{\prime} \overline{\mathrm{G}}_{42} \overline{\mathrm{G}}_{\mathrm{k} 2}+2 \overline{\mathrm{a}}_{66}^{\prime} \overline{\mathrm{G}}_{43} \overline{\mathrm{G}}_{\mathrm{k} 3}\right. \\
& +2 \overline{\mathrm{a}}_{12}^{\prime} \overline{\mathrm{G}}_{42} \overline{\mathrm{G}}_{\mathrm{k} 1}+2 \overline{\mathrm{a}}_{12}^{\prime} \overline{\mathrm{G}}_{41} \overline{\mathrm{G}}_{\mathrm{k} 2}+2 \overline{\mathrm{a}}_{16}^{\prime} \overline{\mathrm{G}}_{43} \overline{\mathrm{G}}_{\mathrm{k} 1}+2 \overline{\mathrm{a}}_{16}^{\prime} \overline{\mathrm{G}}_{41} \overline{\mathrm{G}}_{\mathrm{k} 3}+2 \overline{\mathrm{a}}_{26}^{\prime} \overline{\mathrm{G}}_{43} \overline{\mathrm{G}}_{\mathrm{k} 2} \\
& \left.+2 \overline{\mathrm{a}}_{26}^{\prime} \overline{\mathrm{G}}_{42} \overline{\mathrm{G}}_{\mathrm{k} 3}\right] \mathrm{d} \mathrm{A}+\alpha_{5} \int_{\mathrm{A}}\left[2 \overline{\mathrm{a}}_{11}^{\prime} \overline{\mathrm{G}}_{51} \overline{\mathrm{G}}_{\mathrm{k} 1}+2 \overline{\mathrm{a}}_{22}^{\prime} \overline{\mathrm{G}}_{52} \overline{\mathrm{G}}_{\mathrm{k} 2}+2 \overline{\mathrm{a}}_{66}^{\prime} \overline{\mathrm{G}}_{53} \overline{\mathrm{G}}_{\mathrm{k} 3}\right. \\
& +2 \overline{\mathrm{a}}_{12}^{\prime} \overline{\mathrm{G}}_{52} \overline{\mathrm{G}}_{\mathrm{k} 1}+2 \overline{\mathrm{a}}_{12}^{\prime} \overline{\mathrm{G}}_{51} \overline{\mathrm{G}}_{\mathrm{k} 2}+2 \overline{\mathrm{a}}_{16}^{\prime} \overline{\mathrm{G}}_{53} \overline{\mathrm{G}}_{\mathrm{k} 1}+2 \overline{\mathrm{a}}_{16}^{\prime} \overline{\mathrm{G}}_{51} \overline{\mathrm{G}}_{\mathrm{k} 3}+2 \overline{\mathrm{a}}_{26}^{\prime} \overline{\mathrm{G}}_{53} \overline{\mathrm{G}}_{\mathrm{k} 2} \\
& \left.+2 \overline{\mathrm{a}}_{26}^{\prime} \overline{\mathrm{G}}_{52} \overline{\mathrm{G}}_{\mathrm{k} 3}\right] \mathrm{d} \mathrm{A}+\alpha_{6} \int_{\mathrm{A}}\left[2 \overline{\mathrm{a}}_{11}^{\prime} \overline{\mathrm{G}}_{61} \overline{\mathrm{G}}_{\mathrm{k} 1}+2 \overline{\mathrm{a}}_{22}^{\prime} \overline{\mathrm{G}}_{62} \overline{\mathrm{G}}_{\mathrm{k} 2}+2 \overline{\mathrm{a}}_{66}^{\prime} \overline{\mathrm{G}}_{63} \overline{\mathrm{G}}_{\mathrm{k} 3}\right. \\
& +2 \overline{\mathrm{a}}_{12}^{\prime} \overline{\mathrm{G}}_{62} \overline{\mathrm{G}}_{\mathrm{k} 1}+2 \overline{\mathrm{a}}_{12}^{\prime} \overline{\mathrm{G}}_{61} \overline{\mathrm{G}}_{\mathrm{k} 2}+2 \overline{\mathrm{a}}_{16}^{\prime} \overline{\mathrm{G}}_{63} \overline{\mathrm{G}}_{\mathrm{k} 1}+2 \overline{\mathrm{a}}_{16}^{\prime} \overline{\mathrm{G}}_{61} \overline{\mathrm{G}}_{\mathrm{k} 3}+2 \overline{\mathrm{a}}_{26}^{\prime} \overline{\mathrm{G}}_{63} \overline{\mathrm{G}}_{\mathrm{k} 2} \\
& \left.+2 \overline{\mathrm{a}}_{26}^{\prime} \overline{\mathrm{G}}_{62} \overline{\mathrm{G}}_{\mathrm{k} 3}\right] \mathrm{dA} \\
& =-\int_{A}\left[2 \overline{\mathrm{a}}_{11}^{\prime} \mathrm{c}_{1} \overline{\mathrm{G}}_{\mathrm{k} 1}+2 \overline{\mathrm{a}}_{22}^{\prime} \mathrm{c}_{2} \overline{\mathrm{G}}_{\mathrm{k} 2}+2 \overline{\mathrm{a}}_{12}^{\prime} \mathrm{c}_{2} \overline{\mathrm{G}}_{\mathrm{k} 1}+2 \overline{\mathrm{a}}_{12}^{\prime} \mathrm{c}_{1} \overline{\mathrm{G}}_{\mathrm{k} 2}+2 \overline{\mathrm{a}}_{16}^{\prime} \mathrm{c}_{1} \overline{\mathrm{G}}_{\mathrm{k} 3}\right. \\
& \left.+2 \overline{\mathrm{a}}_{26}^{\prime} \mathrm{c}_{2} \overline{\mathrm{G}}_{\mathrm{k} 3}\right] \mathrm{d} \mathrm{A} \text {. }
\end{aligned}
$$

A two point Gauss integration formula computed the plane integrals. The domain of integration was represented as a rectangular area where the circular 
variable is in domain $[0,2 \pi]$ and radial variable is in domain $[a, b]$. The number of sub-sections in the integrated area has a strong effect on the accuracy of the final result. Here 60 sub-sections were enough to reach the required accuracy.

By solving the system (18) for unknown coefficients $\alpha_{1}, \alpha_{2}, \ldots, \alpha_{6}$, the Airy function is completed. Substituting the Airy stress function into Equation (10) and Equation (11), the elements of the stress and strain tensors are determined. Displacements $\mathfrak{u}_{r}$ and $\mathfrak{u}_{\varphi}$ can be determined from Equation (10) but two additional conditions must be fulfilled: $\mathfrak{u}_{r}(r, 0)=u_{r}(r, 2 \pi)$ and $\mathfrak{u}_{\varphi}(\mathrm{r}, 0)=\mathfrak{u}_{\varphi}(\mathrm{r}, 2 \pi)$.

\section{$5 \quad$ Numerical results}

Numerical evaluation of stress, strain and displacement states in the circular annulus has been made for an orthotropic material. The material is steel sheet type M800-65D, designation EN 10027-1. This material is used as electrical steel sheet in the production of rotor and stator lamellas for electric motors. The material was subjected to a tensile test in order to define its Cartesian orthotropy. From an extremely large database of material properties only the Young's modulus and Poisson's ratio are relevant for calculations.

Two directions, known in steel sheet production, are taken as the principle axes of the Cartesian coordinate system: $x_{1}$ is the axis in the direction of rolling; and $x_{2}$ is the axis perpendicular to $x_{1}$.

\section{Orthotropic steel sheet data}

$$
\begin{aligned}
& p_{\mathrm{n}}=50 \mathrm{~N} / \mathrm{mm}^{2}, \quad \mathrm{a}=20 \mathrm{~mm}, \quad \mathrm{E}_{1}=150320 \mathrm{~N} / \mathrm{mm}^{2}, \\
& \mathrm{p}_{z}=-30 \mathrm{~N} / \mathrm{mm}^{2}, \quad \mathrm{~b}=40 \mathrm{~mm}, \quad \mathrm{E}_{2}=205120 \mathrm{~N} / \mathrm{mm}^{2}, \\
& \mathrm{G}_{12}=62300 \mathrm{~N} / \mathrm{mm}^{2}, \quad v_{12}=0.34 .
\end{aligned}
$$




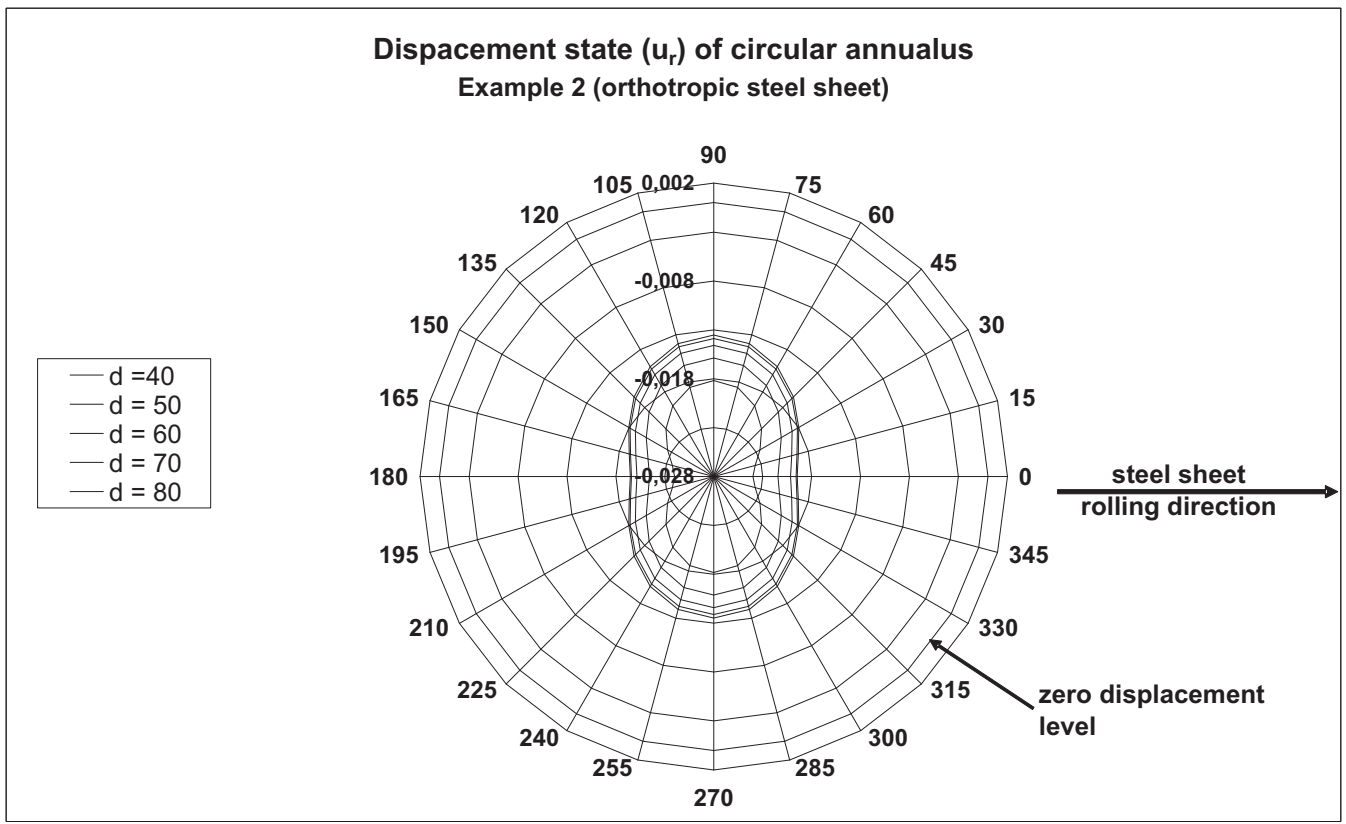

FiguRE 2: Displacement state $\boldsymbol{u}_{\mathrm{r}}$.

The shear modulus $\mathrm{G}_{12}$ is determined in the steel sheet plane $\left(\mathrm{x}_{1}, \mathrm{x}_{2}\right)$ using the relation

$$
\frac{1}{\mathrm{G}_{12}}=\left(\frac{1+v_{12}}{\mathrm{E}_{1}}+\frac{1+v_{21}}{\mathrm{E}_{2}}\right),
$$

where $\boldsymbol{v}_{21}$ is computed from Equation (4). Table 1 list numerically determined results of the orthotropic circular annulus for different radii $\mathrm{R}=$ 20, 30, $40 \mathrm{~mm}$.

Very small deviations of the stress state $\sigma_{\mathrm{r}}$ with regard to changing coordinate $\varphi$ can be noticed in Table 1 . Many more deviations in the distribution of stress state $\sigma_{\varphi}$ can also be seen. Additionally, shear stress $\tau_{r}$ occurs inside the circular annulus. In comparison to $\sigma_{\mathrm{r}}$ in $\sigma_{\varphi}$ stress values, the shear stress $\tau_{r \varphi}$ is relatively smaller by a factor of a thousand. 
TABLE 1: Numerical results for orthotropic material.

\begin{tabular}{|c|c|c|c|c|c|c|}
\hline $\begin{array}{l}\mathrm{R} \\
{[\mathrm{mm}]}\end{array}$ & $\begin{array}{l}\varphi \\
{\left[{ }^{\circ}\right]}\end{array}$ & $\begin{array}{l}\sigma_{\mathrm{r}} \\
{\left[\mathrm{N} / \mathrm{mm}^{2}\right]}\end{array}$ & $\begin{array}{l}\sigma_{\varphi} \\
{\left[\mathrm{N} / \mathrm{mm}^{2}\right]}\end{array}$ & $\begin{array}{l}\tau_{\mathrm{r} \varphi} \\
{\left[\mathrm{N} / \mathrm{mm}^{2}\right]}\end{array}$ & $\begin{array}{l}\mathrm{u}_{\mathrm{r}} \cdot 10^{-2} \\
{[\mathrm{~mm}]}\end{array}$ & $\begin{array}{l}\mathrm{u}_{\varphi} \cdot 10^{-2} \\
{[\mathrm{~mm}]}\end{array}$ \\
\hline \multirow{13}{*}{$20=a$} & 0 & 50 & -163.16 & 0 & -2.4 & 0 \\
\hline & 30 & 50 & -163.25 & 0 & -2.22 & 0.252 \\
\hline & 60 & 50 & -163.41 & 0 & -1.96 & 0.252 \\
\hline & 90 & 50 & -163.49 & 0 & -1.81 & 0 \\
\hline & 120 & 50 & -163.41 & 0 & -1.96 & -0.252 \\
\hline & 150 & 50 & -163.25 & 0 & -2.25 & -0.252 \\
\hline & 180 & 50 & -163.16 & 0 & -2.4 & 0 \\
\hline & 210 & 50 & -163.25 & 0 & -2.25 & 0.252 \\
\hline & 240 & 50 & -163.41 & 0 & -1.96 & 0.252 \\
\hline & 270 & 50 & -163.49 & 0 & -1.81 & 0 \\
\hline & 300 & 50 & -163.41 & 0 & -1.96 & -0.252 \\
\hline & 330 & 50 & -163.25 & 0 & -2.25 & -0.252 \\
\hline & 360 & 50 & -163.16 & 0 & -2.4 & 0 \\
\hline \multirow[t]{13}{*}{30} & 0 & -9.26 & -104.31 & 0 & -2.01 & 0 \\
\hline & 30 & -9.25 & -104.19 & 0.064 & -1.87 & 0.238 \\
\hline & 60 & -9.25 & -103.95 & 0.049 & -1.59 & 0.238 \\
\hline & 90 & -9.26 & -103.83 & 0 & -1.45 & 0 \\
\hline & 120 & -9.25 & -103.95 & -0.049 & -1.59 & -0.238 \\
\hline & 150 & -9.25 & -104.19 & -0.064 & -1.87 & -0.238 \\
\hline & 180 & -9.26 & -104.31 & 0 & -2.01 & 0 \\
\hline & 210 & -9.25 & -104.19 & 0.064 & -1.87 & 0.238 \\
\hline & 240 & -9.25 & -103.95 & 0.049 & -1.59 & 0.238 \\
\hline & 270 & -9.26 & -103.83 & 0 & -1.45 & 0 \\
\hline & 300 & -9.25 & -103.95 & -0.049 & -1.59 & -0.238 \\
\hline & 330 & -9.25 & -104.19 & -0.064 & -1.87 & -0.238 \\
\hline & 360 & -9.26 & -104.31 & 0 & -2.01 & 0 \\
\hline \multirow[t]{13}{*}{$40=b$} & 0 & -30 & -80.53 & 0 & -1.94 & 0 \\
\hline & 30 & -30 & -82.13 & 0 & -1.79 & 0.282 \\
\hline & 60 & -30 & -84.71 & 0 & -1.50 & 0.282 \\
\hline & 90 & -30 & -85.75 & 0 & -1.35 & 0 \\
\hline & 120 & -30 & -84.71 & 0 & -1.50 & -0.282 \\
\hline & 150 & -30 & -82.13 & 0 & -1.79 & -0.282 \\
\hline & 180 & -30 & -80.53 & 0 & -1.94 & 0 \\
\hline & 210 & -30 & -82.13 & 0 & -1.79 & 0.282 \\
\hline & 240 & -30 & -84.71 & 0 & -1.50 & 0.282 \\
\hline & 270 & -30 & -85.75 & 0 & -1.35 & 0 \\
\hline & 300 & -30 & -84.71 & 0 & -1.50 & -0.282 \\
\hline & 330 & -30 & -82.13 & 0 & -1.79 & -0.282 \\
\hline & 360 & -30 & -80.53 & 0 & -1.94 & 0 \\
\hline
\end{tabular}




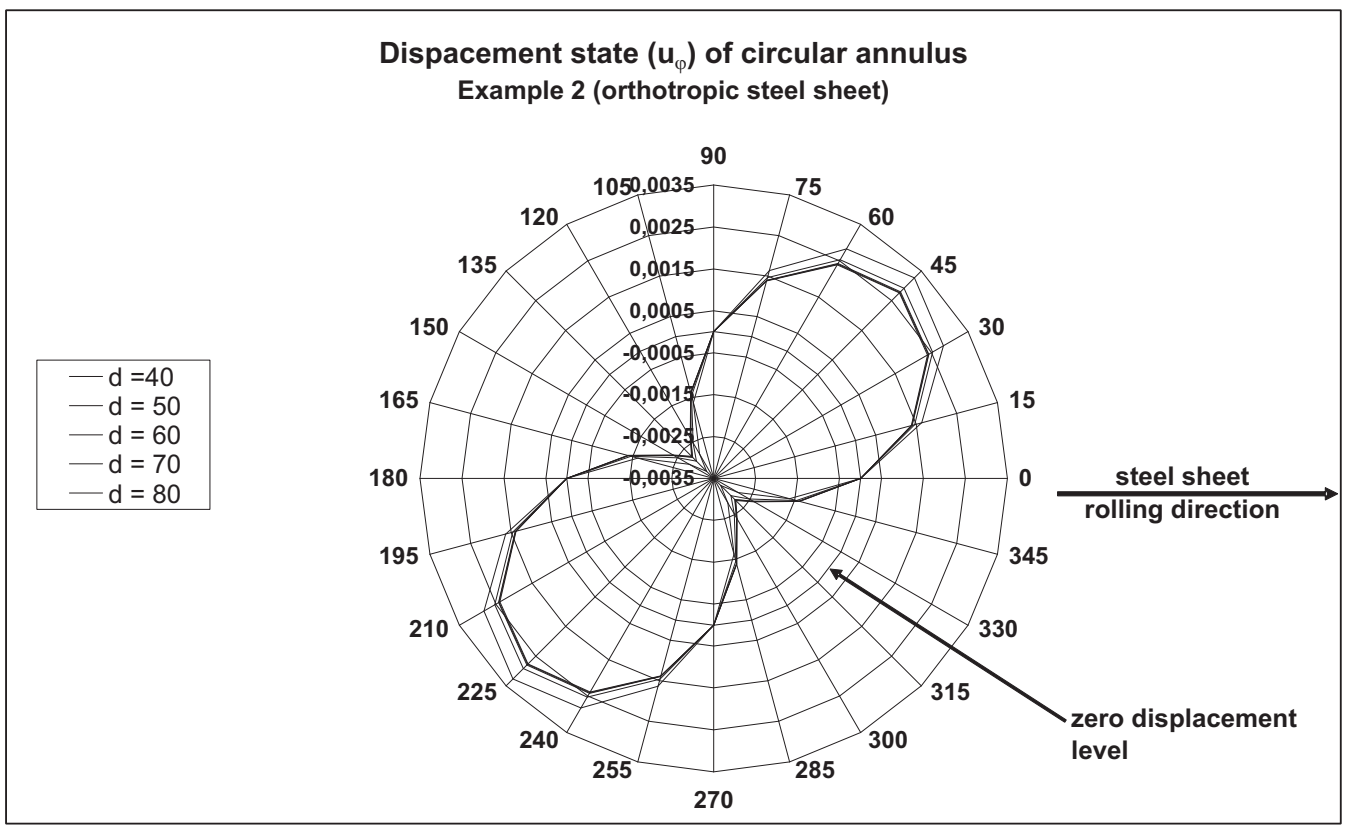

Figure 3: Displacement state $\mathfrak{u}_{\varphi}$. 
Displacements $\mathfrak{u}_{r}$ and $\mathfrak{u}_{\varphi}$ are presented on Figures 2 and 3. The radial displacement $\mathfrak{u}_{r}$ is symmetrical in reference to the rolling direction, axis $x_{1}$, as well as in reference to the perpendicular direction, axis $\boldsymbol{x}_{2}$, Figure 2. The largest radial displacement is observed in the direction of rolling. This result is in agreement with experience and observation in high volume production of components having the shape of a circular annulus.

Figure 3 shows the displacement state in the circular direction $\mathfrak{u}_{\varphi}$. It is symmetric in reference to geometrical lines $x_{1}=x_{2}$ and $x_{1}=-x_{2}$. Regardless of the direction of displacements, the largest are located at $45^{\circ}$ and $135^{\circ}$.

Some additional simulations have shown a high influence of shear modulus value on the form of $\mathfrak{u}_{\varphi}$ displacement state. In comparison with $\mathfrak{u}_{r}$, displacements $\mathfrak{u}_{\varphi}$ are approximately ten times smaller.

\section{Conclusion}

With today's technology in steel sheet production it is not possible to produce an isotropic material. The steel working industry has to deal with experimentally tested orthotropic steel sheet material where the degree of orthotropy is defined by relatively simple experimental measurement methods.

Cartesian orthotropy can be accurately described without any complicated investigation by a uniaxial tensile test.

The Airy stress function originally contains an infinite number of terms which consist of the trigonometric function and polynomial. High convergence is observed by taking only several terms into consideration. This easily yields the required accuracy for algorithms.

All integrals were solved analytically. The choice of the appropriate number of sub-sections inside two point Gauss integration formula has been confirmed to achieve the required accuracy. 
In the numerical case, the input data of Cartesian orthotropy of the M800$65 \mathrm{D}$ steel sheet were determined experimentally. Numerical results show that orthotropy has a big influence especially on displacement in the radial direction. The axis of displacement symmetry in the radial direction and axis of displacement symmetry in the circular direction are rotated one to another by $45^{\circ}$. The same conclusion could be made for the stress state analysis.

\section{References}

[1] F. Kosel. The stress and deflection state in the circular annulus with Cartesian orthotropy. Proceedings 14th Canadian Congress of Applied Mechanics, volume 2, pages 493-494. CANCAM, 1993. C1133, C1138

[2] Pistonesi, C. and Laura, P. A. A., Forced vibrations of a clamped, circular plate of rectangular orthotropy, J. Sound Vibr., 228, 1999, 712-716. C1133

[3] Tahan, N., Pavlovi, M. N. and Kotsovos, M. D., Orthotropic rectangular plates under in-plane loading part 1: closed-form solutions for stresses, Compos. Struct., 33, 1995, 35-48. C1133

[4] Wu, Z. J. and Wardenier, J., Further investigation on the exact elasticity solution for anisotropic thick rectangular plates, Int. J. Solids Struct., 35, 1998, 747-758. C1133

[5] Adewale, A. O., Application of the singularity function method to semi-infinite orthotropic rectangular plates on an elastic foundation, Int. J. Mech. Sci., 43, 2001, 2261-2279. C1133

[6] Bhaskar, K. and Kaushik, B., Simple and exact series solutions for flexure of orthotropic rectangular plates with any combination of clamped and simply supported edges, Compos. Struct., 63, 2004, 63-68. C1133 
[7] S. G. Lekhnitskii. Theory of elasticity of an anisotropic body. Mir Publishers, Moscow, 1981. C1133, C1135, C1136

\section{Author addresses}

1. F. Kosel, University of Ljubljana, Faculty of Mechanical engineering, Aškerčeva 6, 1000 Ljubljana, Slovenia.

2. V. Bratuš, Rotomatika d.o.o., Sp. Kanomlja 23, 5281, Sp. Idrija, Slovenia.

3. T. Kosel, University of Ljubljana, Faculty of Mechanical engineering, Aškerčeva 6, 1000 Ljubljana, Slovenia.

4. T. Videnič, University of Ljubljana, Faculty of Mechanical engineering, Aškerčeva 6, 1000 Ljubljana, Slovenia. mailto:tomaz.videnic@fs.uni-lj.si 